\title{
TRANSPORT OF POLLUTANT IN SHALLOW WATER USING KINETIC SCHEMES
}

\author{
MARIE-OdILE BRISTEAU ${ }^{1}$ AND Benô̂T PERTHAME ${ }^{1,2}$
}

\begin{abstract}
We consider the Saint-Venant system for Shallow Water which is an usual model to describe the flows in rivers or coastal areas. We add to this system an equation modeling the transport of a pollutant or of temperature. The hyperbolic system of conservation laws is solved on unstructured meshes using a finite volume method together with a kinetic scheme. A useful property of this conservative scheme is the built-in preservation of the water depth positivity when applications with dry areas are considered. In this paper we show how the additional transport equation can be discretized in preserving the conservation of pollutant, the monotonicity of its concentration and in keeping it at rest when the computed flow velocity vanishes. Some numerical results illustrate the ability of the method to treat complex problems.
\end{abstract}

AMS Subject Classification. 65C20, 65M60, 76M25.

\section{INTRODUCTION}

We consider the transport of a pollutant or of temperature by shallow water. The flow is classically modeled using a system introduced by Saint-Venant ([24], p. 153) and concerns rivers, lakes or coastal areas. We treat the conservative form of the Saint-Venant equations which are written as a first order hyperbolic system with sources coming from the bottom topography for instance. There are several difficulties encountered in the numerical solution of the problem. For the flow field itself, the conservation of water and the positivity of water depth, when applications with dry areas are considered, should be ensured and the numerical solution should preserve the steady state of still water where the flow velocity vanishes and the water level is constant, therefore the water depth can vary depending on the bottom. For the pollutant, one wishes also to preserve its total mass and also the monotonicity of its concentration; for the still water the pollutant should also be at rest.

A classical approach for solving multidimensional hyperbolic systems consists in using finite volume schemes, see Godlewski and Raviart [10], LeVeque [16], Eymard, Gallouët and Herbin [6]. This method can be used on general triangular grids with a finite element data structure using a particular control volume which is the mediane based dual cell as it was introduced several years ago for aerodynamics problems (see [1] for instance). Let us recall that finite volume methods require to interpolate fluxes at the control volumes interfaces, and the overall stability of the method (and more precisely the in cell entropy property) requires some upwinding in the

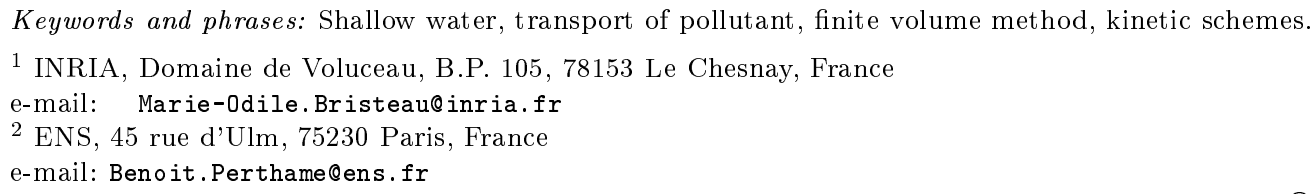


interpolation of the fluxes, this is usually called the 'building block' or 'approximate Riemann solver'. Among those solvers, the original Godunov solver is particularly noticeable, also a very good and popular compromise between stability, accuracy and numerical efficiency is the so-called Roe solver (see [22], [10] for instance). The difficulty to understand the convergence of numerical schemes is related to the deep mathematical structure of such hyperbolic systems; the first existence proof of weak solutions after shocks in the large is due to [18] in 1995. It is based on the kinetic interpretation of the system which is also a method to derive numerical schemes with rigorous stability, accuracy and numerical efficiency properties (see [20]). In this paper we describe such a kinetic solver applied to the Saint-Venant system and especially for the case with transport of a pollutant.

Concerning this system, another well known difficulty is, as mentionned above, that the scheme preserves steady states like that given by still water. This difficulty related to the source term due to bottom slope has been treated by several authors along with an early idea of Roe [23] to upwind the source terms at the interfaces. Bermudez and Vasquez [4], Bermudez et al [3] propose to realize the steady states at high enough order. The computation of exact steady states has been performed by Greenberg and Leroux [13], Gosse and Leroux [12], Gosse [11], Godinaud et al [9], especially for Godunov solver and using the concept of 'well-balanced' scheme. Together with Roe solver, improvments and simplifications have also been proposed recently by Gallouët, Hérard and Seguin [7]. Another method, based on introducing a discontinuity in the computational cells is proposed by LeVeque [17], and Jin [15] proposes to realize the steady states at the interfaces rather than at the cell centers. Concerning the kinetic solver that we discuss here, the problem to preserve the steady states has been treated in [2] and the method has given rise to the numerical code that we use here. For the simpler case of a scalar conservation law, a kinetic scheme that preserves equilibrium and which is proved to be convergent, is presented in Botchorishvili et al [5]. The idea here is that, at the microscopic level, the topography acts like a potential for classical particles, and they can be either reflected or transmitted with an energy jump through the potential discontinuity. This approach is extended to the shallow water case in [21].

After presenting the equations in Section 2, we introduce in Section 3 the kinetic formulation and we deduce a kinetic scheme in Section 4. In section 5, we present some properties of the scheme and in Section 6 we illustrate the possibilities of the method by numerical results.

\section{EquATIONS}

We consider the 2D Saint-Venant system, written in its physical conservative form (see Gerbeau and Perthame [8] for a derivation departing from the Navier-Stokes system),

$$
\begin{gathered}
\frac{\partial h}{\partial t}+\operatorname{div}(h u)=S, \\
\frac{\partial h u}{\partial t}+\operatorname{div}(h u \otimes u)+\nabla\left(\frac{g}{2} h^{2}\right)+g h \nabla Z=0,
\end{gathered}
$$

where we denote $h(t, x) \geq 0$, the water depth, $u(t, x) \in \mathbb{R}^{2}$, the flow velocity, $g$ the acceleration due to gravity intensity and $Z(x)$ the bottom height, and therefore $h+Z$ is the level of water surface (see Fig. 2.1). We denote also $q(t, x)=h(t, x) u(t, x)$, the flux of water and $\mathrm{S}(\mathrm{t}, \mathrm{x})$ is the given source term which can contain the pollutant.

We add to this system, an equation for the advection of a pollutant concentration $\mathrm{T}(\mathrm{t}, \mathrm{x})$ (or temperature). This decoupled equation is written in a conservative form as

$$
\frac{\partial h T}{\partial t}+\operatorname{div}(h u T)=T_{s} S
$$




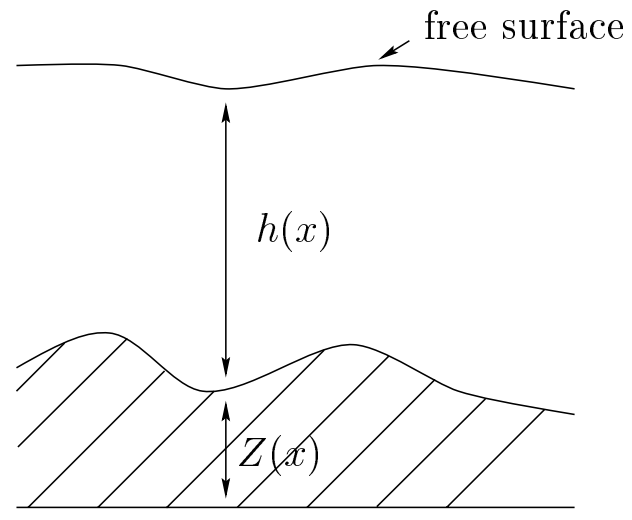

Figure 2.1:

but 'monotonicity' is better seen on the developed form

$$
\frac{\partial T}{\partial t}+u \cdot \nabla T=0
$$

In the equation (2.3), $T_{s}$ is the given value of the pollutant concentration (or temperature) at the source $\mathrm{S}$. We will denote also $e=h T$. The pollutant may also be introduced in the flow by the initial or the boundary conditions.

This system of equations can be written as a system of first order conservation laws

$$
\frac{\partial U}{\partial t}+\operatorname{div} F(U)=B(U)
$$

with

$$
\begin{gathered}
U=\left(\begin{array}{c}
h \\
q \\
e
\end{array}\right), \quad F(U)=\left(\begin{array}{c}
q \otimes q \\
h \\
\frac{q e^{2}}{h}
\end{array}\right), \\
B(U)=\left(\begin{array}{c}
g \\
-g h \nabla Z \\
T_{s} S
\end{array}\right) .
\end{gathered}
$$

\section{KinetiC APPROACH}

We introduce a kinetic approach to system (2.1)-(2.3) written with $S=0$. Then we can deduce from the discretization of the kinetic equations, a kinetic scheme for the initial system.

Let $\chi(w)$ be a positive, even function defined on $\mathbb{R}^{2}$ and satisfying

$$
\int_{\mathbb{R}^{2}}\left(\begin{array}{c}
1 \\
w_{i} w_{j}
\end{array}\right) \chi(w) d w=\left(\begin{array}{c}
1 \\
\delta_{i j}
\end{array}\right) .
$$

In addition we assume that $\chi(w)$ is compactly supported, i.e.

$$
\exists w_{M} \in \mathbb{R} \text {, such that } \chi(w)=0 \text { for }|w| \geq w_{M} .
$$


An example of function $\chi$ satisfying these properties is

$$
\chi(w)=\frac{1}{12} \mathbb{I}_{\left|w_{i}\right| \leq \sqrt{3}}, \quad i=1,2 .
$$

We introduce two microscopic densities of particles $M(t, x, \xi)$ and $N(t, x, \xi)$ defined by a so-called Gibbs equilibrium

$$
\begin{aligned}
& M(t, x, \xi)=\frac{h(t, x)}{\tilde{c}^{2}} \chi\left(\frac{\xi-u(t, x)}{\tilde{c}}\right), \\
& N(t, x, \xi)=\frac{h T(t, x)}{\tilde{c}^{2}} \chi\left(\frac{\xi-u(t, x)}{\tilde{c}}\right)
\end{aligned}
$$

with $\tilde{c}$ defined by

$$
\tilde{c}^{2}=\frac{g h}{2}
$$

We will denote

$$
G(t, x, \xi)=\left(\begin{array}{c}
M(t, x, \xi) \\
N(t, x, \xi)
\end{array}\right)
$$

With these definitions we can write a kinetic interpretation of the system (2.1)-(2.3) and we have the following statement:

Theorem 3.1. The functions $(h, q, e)$ are weak solutions to the system (2.1)-(2.3) or (2.5)-(2.7) if and only if $G(t, x, \xi)$ satisfies the kinetic equation

$$
\frac{\partial G}{\partial t}+\xi \cdot \nabla_{x} G-g \nabla Z \cdot \nabla_{\xi} G=Q(t, x, \xi)
$$

for some "collision terms" $Q(t, x, \xi)=\left(\begin{array}{l}Q_{1}(t, x, \xi) \\ Q_{2}(t, x, \xi)\end{array}\right)$ which satisfy for a.e. $(t, x)$,

$$
\int_{\mathbb{R}^{2}}\left(\begin{array}{c}
1 \\
\xi
\end{array}\right) Q_{1} d \xi=0, \quad \int_{\mathbb{R}^{2}} Q_{2} d \xi=0 .
$$

Notice that the mathematical entropy property, for the energy, can also be considered in terms of the kinetic approach (see [21]).

Proof. The proof relies on a very obvious computation. The two Saint-Venant equations and the pollutant transport equation are equivalent with the equation (3.8) once integrated in $\xi$ against $K(\xi)$ with $K(\xi)$ the following [2x4] matrix

$$
K(\xi)=\left(\begin{array}{cc}
1 & 0 \\
\xi & 0 \\
0 & 1
\end{array}\right) .
$$


These are consequences of the usual relations deduced from the properties of $\chi$ and from (3.9):

$$
\begin{gathered}
\left(\begin{array}{c}
h \\
\frac{q \otimes q}{h}+\frac{g}{2} h^{2} I d
\end{array}\right)=\int_{\mathbb{R}^{2}}\left(\begin{array}{c}
1 \\
\xi \\
\xi \otimes \xi
\end{array}\right) M(\xi) d \xi \\
\left(\begin{array}{c}
e \\
\frac{q e}{h}
\end{array}\right)=\int_{\mathbb{R}^{2}}\left(\begin{array}{c}
1 \\
\xi
\end{array}\right) N(\xi) d \xi
\end{gathered}
$$

and

$$
\left(\begin{array}{c}
0 \\
h \\
0
\end{array}\right)=-\int_{\mathbb{R}^{2}} K(\xi) \nabla_{\xi} G(\xi) d \xi,
$$

This theorem has a very fundamental consequence: the non-linear system (2.1)-(2.3) can be viewed as a system of linear transport equations on nonlinear quantities $M$ and $N$, for which it is easier to find a simple numerical scheme with good theoretical properties.

\section{KinetiC SCHEME}

\subsection{Basic kinetic scheme}

Let $\Omega$ denote the computational domain with boundary $\Gamma$, which we assume polygonal. In order to define the kinetic scheme, we introduce an usual finite volume discretization. Let $\mathcal{T}_{h}$ be a triangulation of $\Omega$ which vertices are denoted $P_{i}$. The dual cells $C_{i}$ are obtained by joining the centers of mass of the triangles surrounding each vertex $P_{i}$. We use the following notations (see Fig. 4.1):

$K_{i}$, set of nodes $P_{j}$ surrounding $P_{i}$,

$\left|C_{i}\right|$, area of $C_{i}$,

$\Gamma_{i j}$, boundary edge belonging to cells $C_{i}$ and $C_{j}$,

$L_{i j}$, length of $\Gamma_{i j}$,

$n_{i j}$, unit normal to $\Gamma_{i j}$, outward to $C_{i}\left(n_{j i}=-n_{i j}\right)$.

Let $\Delta t$ be the timestep, we set $t^{n}=n \Delta t$. We consider the system of equations (2.5) with $B=0$. We refer to [2] for a detailed description of the treatment of term due to the varying bed slope $g h \nabla Z$. We denote by $U_{i}^{n}$ the approximation of the cell average of the exact solution at time $t^{n}$

$$
U_{i}^{n} \simeq \frac{1}{\left|C_{i}\right|} \int_{C_{i}} U\left(t^{n}, x\right) d x
$$

We integrate in space and time the equation (2.5) on the set $C_{i} \times\left(t^{n}, t^{n+1}\right)$, and, integrating by parts the divergence term, we obtain

$$
U_{i}^{n+1}=U_{i}^{n}-\sum_{j \in K_{i}} \sigma_{i j} \mathcal{F}\left(U_{i}^{n}, U_{j}^{n}, n_{i j}\right)
$$

with

$$
\sigma_{i j}=\frac{\Delta t L_{i j}}{\left|C_{i}\right|}
$$




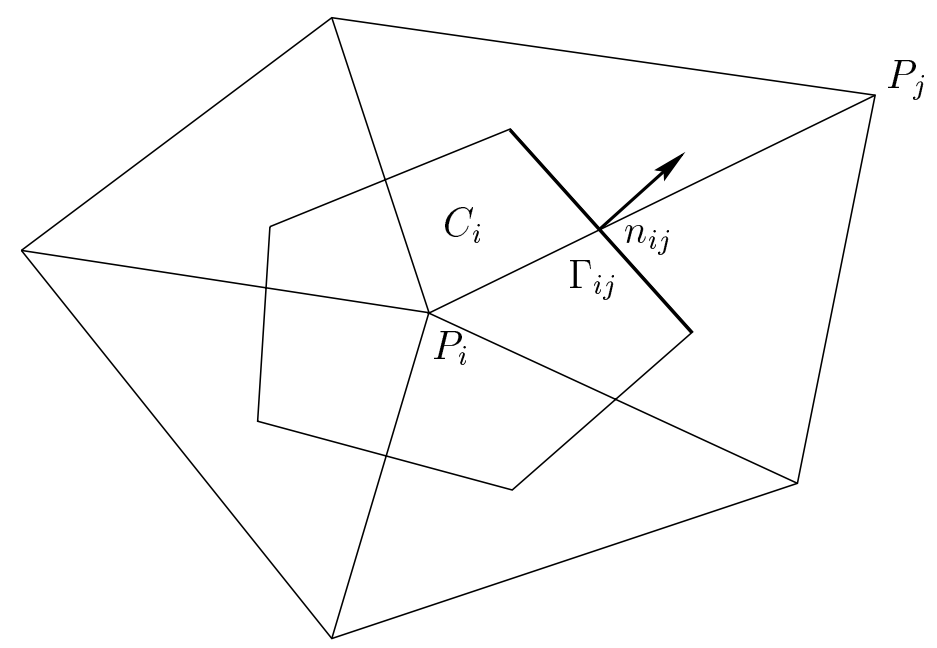

Figure 4.1: Dual cell $C_{i}$.

where $\mathcal{F}\left(U_{i}, U_{j}, n_{i j}\right)$ denotes an interpolation of the normal component of the flux $F(U) \cdot n_{i j}$ along the edge $\Gamma_{i j}$. This interpolation is usually performed using a one dimensional solver since locally the problem looks like a planar discontinuity. Here we define $\mathcal{F}\left(U_{i}, U_{j}, n_{i j}\right)$ using the kinetic interpretation of the system. So, being given the solution $U_{i}^{n}$ at time $t^{n}$ for each cell, we compute $U_{i}^{n+1}$ by the following algorithm with three steps:

- We define $G_{i}^{n}=G\left(h_{i}^{n}, h T_{i}^{n}, \xi-u_{i}^{n}\right)$ with $G$ defined by (3.4)-(3.7).

- We use the microscopic equations (3.8). Since these equations are linear, we can apply a simple upwind scheme which defines a density function $g_{i}^{n+1}(\xi)$

$$
g_{i}^{n+1}(\xi)-G_{i}^{n}(\xi)+\frac{\Delta t}{\left|C_{i}\right|} \sum_{j \in K_{i}} L_{i j} \xi \cdot n_{i j} G_{i j}^{n}(\xi)=0,
$$

with the fluxes $G_{i j}^{n}(\xi)$ computed by the upwind formula

$$
G_{i j}^{n}(\xi)= \begin{cases}G_{i}^{n}(\xi) & \text { for } \xi \cdot n_{i j} \geq 0 \\ G_{j}^{n}(\xi) & \text { for } \xi \cdot n_{i j} \leq 0 .\end{cases}
$$

Notice however that the density function $g(\xi)$ is not an equilibrium (see remark (4.1)).

- Nethertheless, we can recover the macroscopic quantities $U_{i}^{n+1}$ at time $t^{n+1}$ by integration

$$
U_{i}^{n+1}=\int_{\mathbb{R}^{2}} K(\xi) g_{i}^{n+1}(\xi) d \xi .
$$

The numerical feasability of the method relies on the possibility to write directly a finite volume formula, which therefore avoids using the extra variable $\xi$ in the actual implementation. Indeed, the equation (4.5) can be written with the form (4.2) with

$$
\mathcal{F}\left(U_{i}, U_{j}, n_{i j}\right)=F^{+}\left(U_{i}, n_{i j}\right)+F^{-}\left(U_{j}, n_{i j}\right),
$$


and

$$
\begin{aligned}
& F^{+}\left(U_{i}, n_{i j}\right)=\int_{\xi \cdot n_{i j} \geq 0} \xi \cdot n_{i j} K(\xi) G_{i}(\xi) d \xi, \\
& F^{-}\left(U_{j}, n_{i j}\right)=\int_{\xi \cdot n_{i j} \leq 0} \xi \cdot n_{i j} K(\xi) G_{j}(\xi) d \xi .
\end{aligned}
$$

Remark 4.1. The interpretation is that, as usual, the collision terms $Q$, which forces the relaxation of $g$ to Gibbs equilibrium $G$, is neglected in the advection scheme (4.4). And at each timestep we deduce $G_{i}^{n+1}(\xi)$ from $U_{i}^{n+1}$ which is a way to perform all collisions at once and to recover the Gibbs equilibrium without computing it explicitely.

\subsection{Numerical Implementation}

We give here some details on the implementation of the kinetic scheme defined by (4.2), (4.6)-(4.8). For the efficiency of the method, we code in fact a variant where the choice of the function $\chi$ depends on the interface under consideration. For each edge $\Gamma_{i j}$, we define a local basis $\left(x_{1}, x_{2}\right)$ associated to the normal direction and to the tangential one. We denote $\hat{U}$, the vector deduced from $U$ by the rotation in this new basis. If $n_{i j}=\left(n_{x}, n_{y}\right)$, we have $\hat{U}$ defined by

$$
\hat{U}=R U \quad \text { with } \quad R=\left(\begin{array}{cccc}
1 & 0 & 0 & 0 \\
0 & n_{x} & n_{y} & 0 \\
0 & -n_{y} & n_{x} & 0 \\
0 & 0 & 0 & 1
\end{array}\right)
$$

and

$$
F^{+}(U, n)=R^{-1} \hat{F}^{+}(\hat{U}), \quad R^{-1}=\left(\begin{array}{cccc}
1 & 0 & 0 & 0 \\
0 & n_{x} & -n_{y} & 0 \\
0 & n_{y} & n_{x} & 0 \\
0 & 0 & 0 & 1
\end{array}\right) .
$$

Using (4.7), we give the detailed expression of $\hat{F}^{+}\left(\hat{U}_{i}\right)$ related to the edge $\Gamma_{i j}$

$$
\hat{F}^{+}\left(\hat{U}_{i}\right)=\frac{h_{i}}{\tilde{c}_{i}^{2}} \int_{\left(\xi_{1} \geq 0\right) \times \mathbb{R}} \xi_{1}\left(\begin{array}{c}
1 \\
\xi \\
T_{i}
\end{array}\right) \chi\left(\frac{\xi-\hat{u}_{i}}{\tilde{c}_{i}}\right) d \xi
$$

or, after a change of variables,

$$
\hat{F}^{+}\left(\hat{U}_{i}\right)=h_{i} \int_{\left(w_{1} \geq \frac{-\hat{u}_{i, 1}}{\hat{c}_{i}}\right) \times \mathbb{R}}\left(\hat{u}_{i, 1}+w_{1} \tilde{c}_{i}\right)\left(\begin{array}{c}
1 \\
\hat{u}_{i, 1}+w_{1} \tilde{c}_{i} \\
\hat{u}_{i, 2}+w_{2} \tilde{c}_{i} \\
T_{i}
\end{array}\right) \chi(w) d w .
$$

Due to the fact that $\chi(w)$ is even and satisfies (3.1), the term with $w_{2}$ disappears in (4.10) and we obtain the simpler formulas

$$
\hat{F}_{u_{2}}^{+}\left(\hat{U}_{i}\right)=\hat{u}_{i, 2} \hat{F}_{h}^{+}\left(\hat{U}_{i}\right)
$$

and

$$
\hat{F}_{T}^{+}\left(\hat{U}_{i}\right)=T_{i} \hat{F}_{h}^{+}\left(\hat{U}_{i}\right)
$$


We obtain $F^{-}$by an analogous computation and so we have the same property for $\hat{\mathcal{F}}$. We will use this property to deduce a modified scheme with better accuracy. Indeed, when considering steady states for still water, although the flow velocity vanishes, the flux $\hat{F}_{T}$ (a consistent approximation of $h u T$ ) does not vanish. This creates, for long time integration, an important diffusion.

\subsection{Modified scheme}

In order to reduce the diffusion of the scheme, we replace in the equations (4.2) for $u_{2}$ and $T$ the expression of $\hat{\mathcal{F}}$ by the following:

$$
\hat{\mathcal{F}}_{u_{2}}\left(\hat{U}_{i}, \hat{U}_{j}\right)=\hat{u}_{i j, 2} \hat{\mathcal{F}}_{h}\left(\hat{U}_{i}, \hat{U}_{j}\right)
$$

and

$$
\hat{\mathcal{F}}_{T}\left(\hat{U}_{i}, \hat{U}_{j}\right)=T_{i j} \hat{\mathcal{F}}_{h}\left(\hat{U}_{i}, \hat{U}_{j}\right)
$$

with

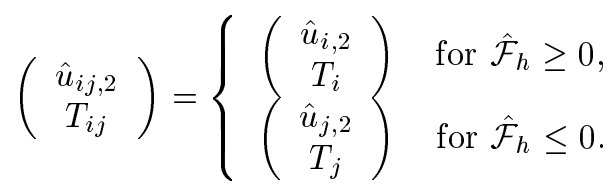

Formula (4.15) introduces some upwinding depending on the sign of the total flux.

One readily checks that the numerical artefact described at the end of the previous subsection has now disappeared. When considering a still water, the flux $\hat{\mathcal{F}}_{h}$ vanishes because this is a steady state, therefore the fluxes $\hat{\mathcal{F}}_{u_{2}}$ and $\hat{\mathcal{F}}_{T}$ also vanish. There is no numerical transport of pollutant.

\section{Properties of the SCHEME}

It is clear from (4.2), (4.6)-(4.8) that the scheme is consistent and conservative. We can also prove (see [2]) that the water depth positivity is preserved under the CFL condition

$$
\Delta t \leq \min \left(\frac{\left|C_{i}\right|}{\left(\sum_{j \in K_{i}} L_{i j}\right)\left(\left|u_{i}^{n}\right|+w_{M} \tilde{c}_{i}^{n}\right)}\right)
$$

and also the monotonicity of the pollutant concentration or of temperature. Especially they cannot become negative or exceed their input values. These stability properties are important when applications with dry areas are considered (see also [2] for examples where this property applies).

The first order scheme defined in the previous section is extended to a "formally" second order one using a MUSCL like extension (see [25]) with a Van Albada type limitor. If this extension is applied directly, the previous stability properties are no more verified, some work is under development on this subject. The following numerical results have been obtained with this second order scheme for the Saint-Venant system but we have used the first order scheme for the pollutant to preserve the monotonicity and particularly the positivity.

\section{Numerical ReSults}

The first test case concerns a partial failure of a dam in a $200 \times 200 \mathrm{~m}$ basin as depicted in Figure 6.1. This problem has been computed by many authors (see e.g. [19] and the references therein). The initial waterheight is $h=10 \mathrm{~m}$ on the left hand side and $h=5 \mathrm{~m}$ on the right of an idealized dam. Water is released into the downstream side through a breach $75 \mathrm{~m}$ wide. The discretization step of the boundaries is $2.5 \mathrm{~m}$, the mesh contains about 8100 nodes and 15800 triangles. In Figure 6.2 we show the watersurface elevation at time $t=7.2$ 
s. The velocity field is plotted in Figure 6.3. On this fine mesh, the vertices on each side of the breach are accurately computed.

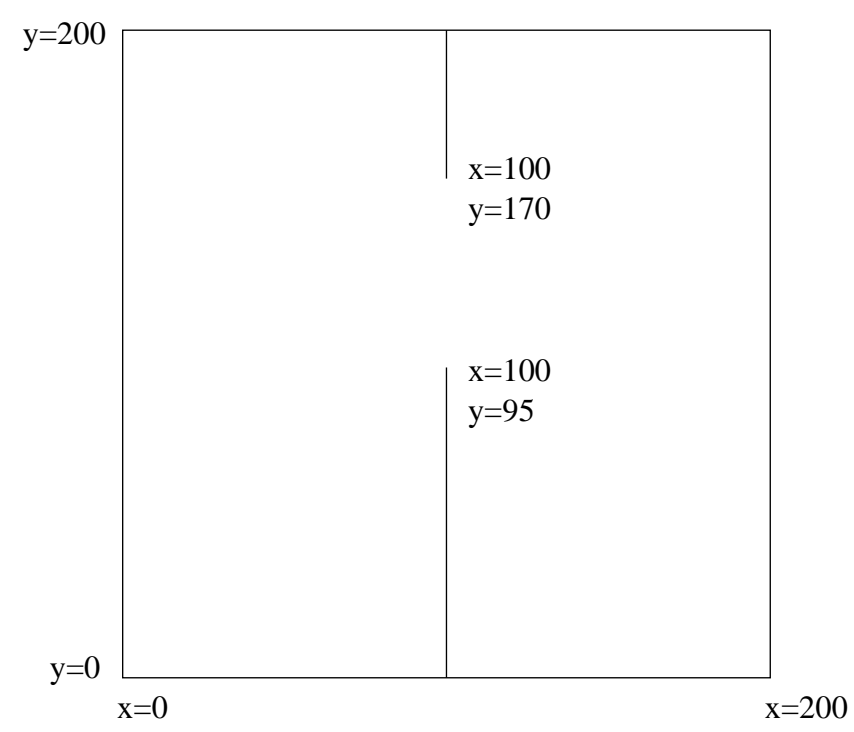

Figure 6.1: Geometry of the basin with dam breach.

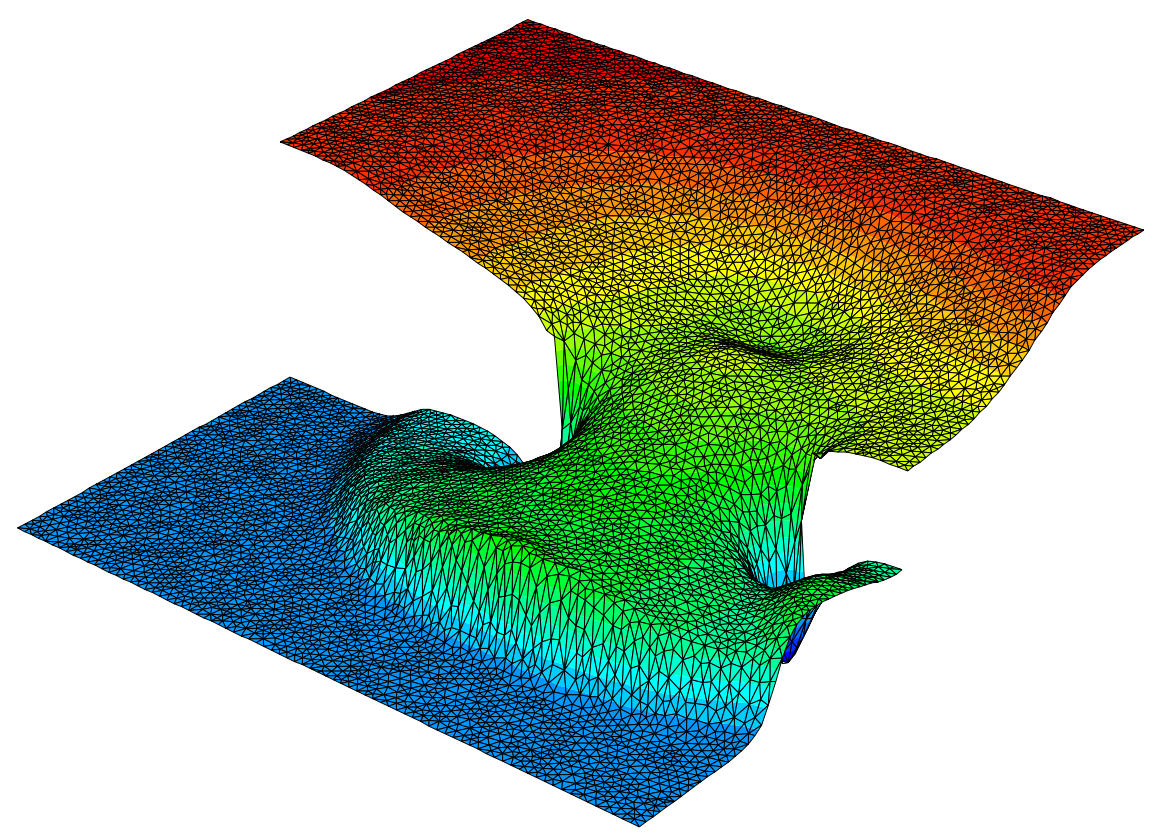

Figure 6.2: Partial dam break: Water elevation at $t=7.2 \mathrm{~s}$. 


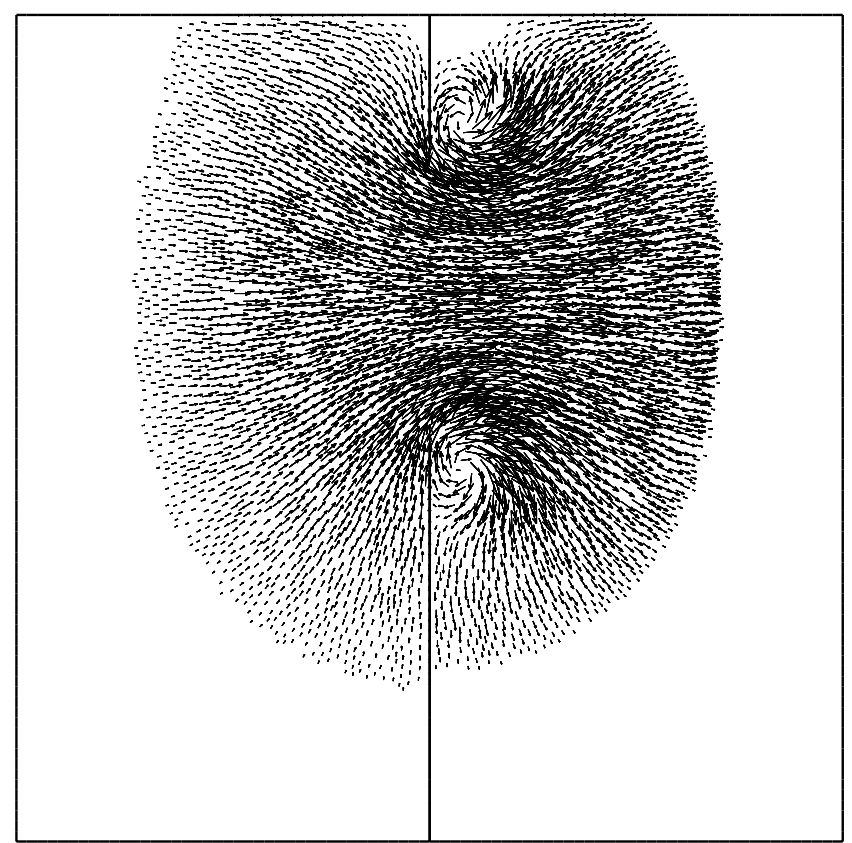

Figure 6.3: Partial dam break: Velocity field at $t=7.2 \mathrm{~s}$.

We also have considered problems on a geometry given as test case in the code Telemac developed at EDF ([14]). It is a river with obstacles, the length is about 1,200 meters, the width $300 \mathrm{~m}$, the bottom is varying from 252 to $260 \mathrm{~m}$ as shown in Figure 6.4. When the water level is more than $260 \mathrm{~m}$ we assume that there are walls on the river sides. The mesh is about 1,000 nodes and 1,850 triangles.

The initial water level is $265 \mathrm{~m}$ and the inflow discharge is growing from 0 . to $500 \mathrm{~m}^{3} / \mathrm{s}$ for $t$ varying from 0 to $1800 \mathrm{~s}$, then the discharge is maintained equal to $500 \mathrm{~m}^{3} / \mathrm{s}$.

On this example we show the effect of the modified scheme introduced in Sec.4.1. In Figure 6.5 (resp. Figure 6.6 we show the velocity field at $t=3600 \mathrm{~s}$ obtained with the scheme defined in Sec.4.1 (resp. Sec.4.3). In Figure 6.6 the vortex is more developed and the solution is in better agreement with solutions obtained by other accurate schemes proposed in Telemac.

We introduce in this flow a pollutant at a source point, we prescribe in equation $(2.1) S=10 \mathrm{~m} / \mathrm{s}$ and in equation (2.3) $T_{s}=10$ for $1000 \mathrm{~s} \leq \mathrm{t} \leq 1800 \mathrm{~s}$ and $3000 \mathrm{~s} \leq \mathrm{t} \leq 3600 \mathrm{~s}$ and $S=0$ otherwise. In Figure 6.7 we present the pollutant concentration at different times. These results have been obtained with the modified scheme of Sec.4.3, the results obtained with the initial scheme are not significant.

\section{Conclusions}

Following the theory of kinetic schemes for gas dynamics ([20]), we have applied these schemes to the SaintVenant system with an additional transport equation. These schemes satisfy built-in stability properties which are essential for the applications. In order to improve the accuracy, we are now working on extensions of the first order schemes to second order ones while preserving the stability properties. Besides, we have not discussed here the treatment of the boundary conditions, it will be done in a forthcoming paper.

The authors would like to thank M. Guesmia, J.M. Hervouet and B. Mohammadi for helpful comments and suggestions. 


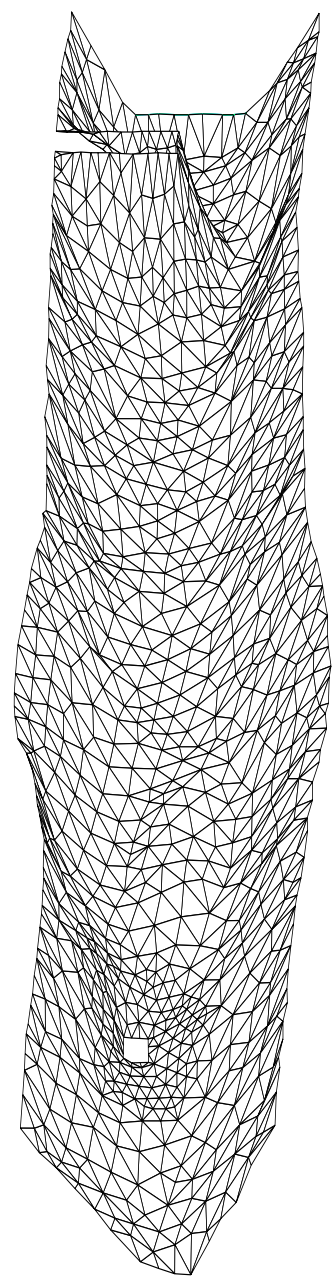

Figure 6.4: River bed

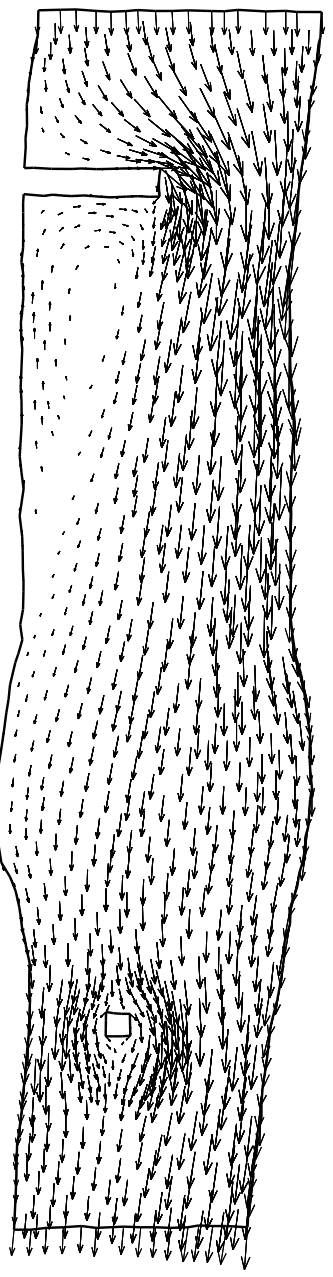

Figure 6.5: Velocity field with initial scheme

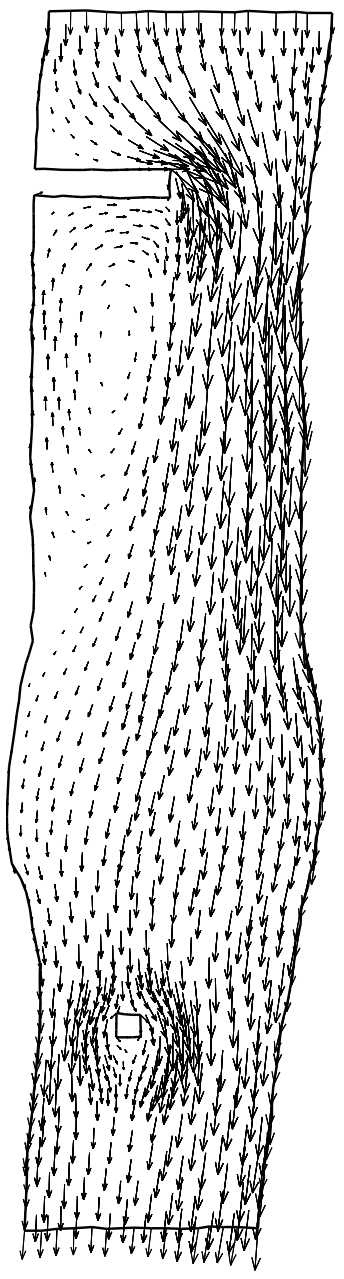

Figure 6.6: Velocity field with modified scheme

\section{REFERENCES}

[1] Angrand F., Dervieux A., Boulard V., Periaux J. and Vijayasundaram G., Transonic Euler simulation by means of finite element explicit schemes, AIAA-83, (1984).

[2] Audusse E., Bristeau,M. O. and Perthame,B., Kinetic schemes for solving Saint-Venant equations with source terms, Inria report, RR-3989 (2000), http://www.inria.fr/RRRT/RR-3989.html

[3] Bermudez A., Dervieux A., Desideri J.A., Vazquez M.E., Upwind schemes for the two-dimensional shallow water equations with variable depth using unstructured meshes, Comput. Methods Appl. Mech. Engrg. 155, no. 1-2, 49-72 (1998).

[4] Bermudez A., Vazquez M.E., Upwind methods for hyperbolic conservation laws with source terms, Comput. Fluids 23, no. 8, 1049-1071 (1994).

[5] Botchorishvili R., Perthame B. and Vasseur A., Equilibrium Schemes for Scalar Conservation Laws with Stiff Sources, Inria report, RR-3891 (2000), http://www.inria.fr/RRRT/RR-3891.html

[6] R. Eymard, T. Gallouët and R. Herbin, Finite volume methods, In Handboook of numerical analysis, vol. VIII, P.G. Ciarlet and J-L. Lions eds., North-Holland, Amsterdam, to appear.

[7] Gallouët T., Hérard J.M. and Seguin N., Some approximate Godunov schemes to compute shallow-water equations with topography, AIAA-2001 (2000). 


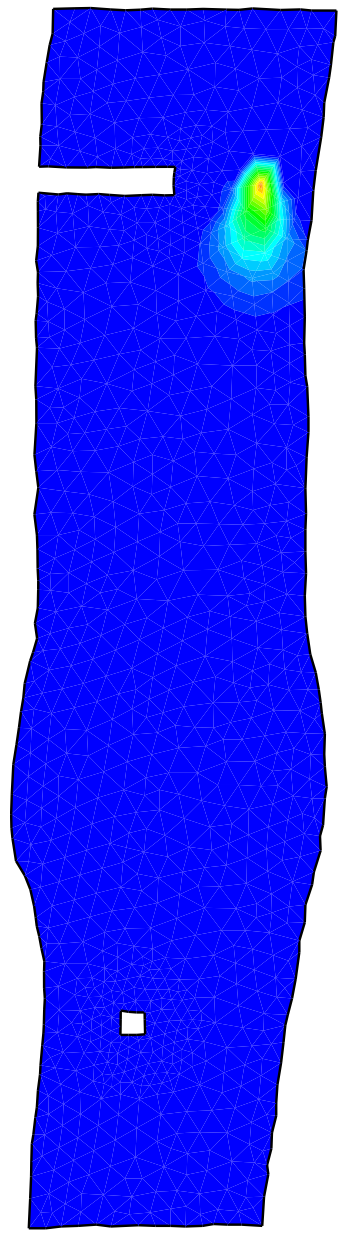

(a) $t=1300 \mathrm{~s}$

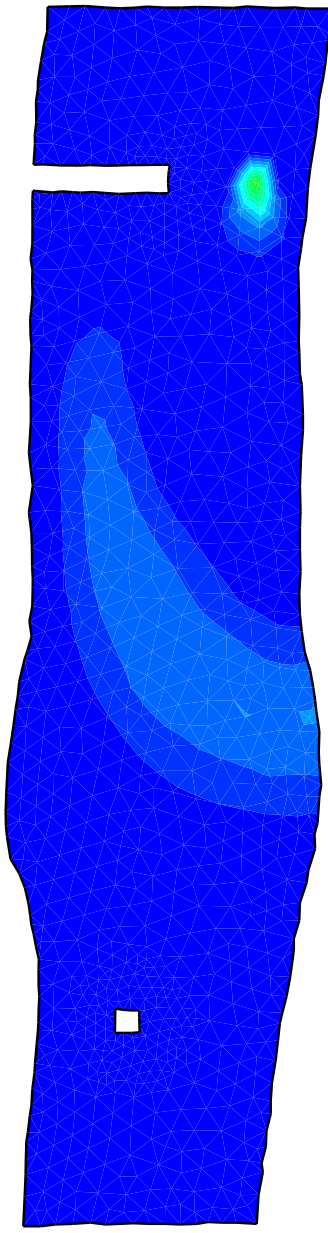

(b) $t=3100 \mathrm{~s}$

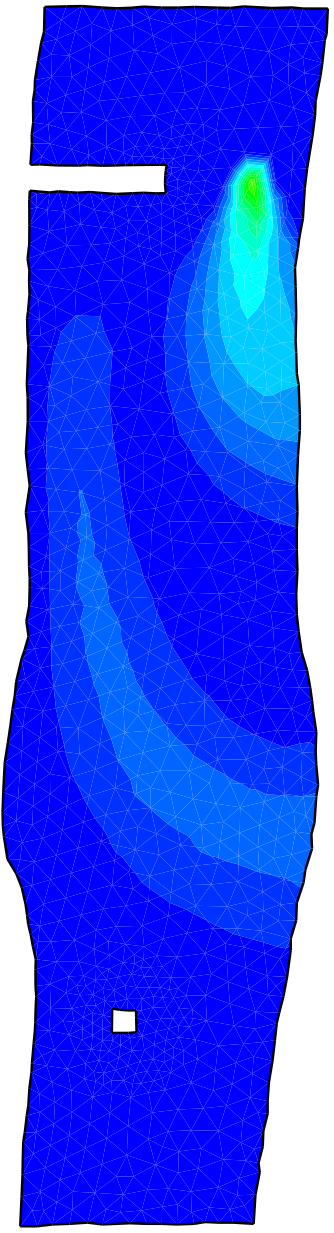

(c) $t=3600 \mathrm{~s}$

Figure 6.7: Pollutant concentration

[8] Gerbeau J.-F. and Perthame B., Derivation of viscous Saint-Venant systems for shallow water or narrow tubes; numerical validation, in preparation.

[9] Godinaud G., LeRoux A.Y., LeRoux M.N., Generation of new solvers involving the source term for a class of nonhomogeneous hyperbolic systems. Preprint.

[10] Godlewski E. and Raviart P.-A., Numerical approximations of hyperbolic systems of conservation laws, Applied Mathematical Sciences 118, Springer-Verlag, New York (1996).

[11] Gosse L., A priori error estimate for a well-balanced scheme designed for inhomogeneous scalar conservation laws, C.R. Acad. Sc. Paris, 327, 467-472 (1998).

[12] Gosse L., Leroux A.-Y., A well-balanced scheme designed for inhomogeneous scalar conservation laws, C. R. Acad. Sc., Paris, Sér. I 323, 543-546 (1996).

[13] Greenberg J.M. and Leroux A.-Y., A well balanced scheme for the numerical processing of source terms in hyperbolic equations, SIAM J. Num. Anal. 33, 1-16 (1996).

[14] Hervouet J.M., TELEMAC modelling system: an overview, Hydrol. Process. 14, 2209-2210, (2000).

[15] Jin S., A steady-states capturing method for hyperbolic systems with source terms, to appear in M2AN. 
[16] LeVeque R.J., Numerical Methods for Conservation Laws, Lectures in Mathematics, ETH Zurich, Birkhauser (1992).

[17] LeVeque R.J., Balancing source terms and flux gradients in high-resolution Godunov methods: the quasi-steady wavepropagation algorithm. J. Comput. Phys. 146, no. 1, 346-365 (1998).

[18] Lions P.L., Perthame B. and Souganidis P.E., Existence of entropy solutions for the hyperbolic systems of isentropic gas dynamics in Eulerian and Lagrangian coordinates, Comm. Pure Appl. Math., 49, no. 6, 599-638 (1996).

[19] Paillère H., Degrez G. and Deconinck H., Multidimensional Upwind schemes for the shallow water equations, Int. J. for Numer. Meth. Fluids, 26, 987-1000, (1998).

[20] Perthame B., An introduction to kinetic schemes for gas dynamics, An introduction to recent developments in theory and numerics for conservation laws. L.N. in Computational Sc. and Eng., 5, D. Kroner, M. Ohlberger and C. Rohde eds, Springer (1998).

[21] Perthame B. and Simeoni C., A kinetic scheme for Saint-Venant equations with a source term, in preparation.

[22] Roe P. L., Approximate Riemann solvers, parameter vectors and difference schemes, J. Comp. Phys. 43, 357-372 (1981).

[23] Roe P. L., Upwind differenced schemes for hyperbolic conservation laws with source terms, Proc. Conf. Hyperbolic Problems, Carasso, Raviart and Serre eds, Springer, 41-51 (1986).

[24] de Saint-Venant A. J. C., Théorie du mouvement non-permanent des eaux, avec application aux crues des rivières et à l'introduction des marées dans leur lit, C. R. Acad. Sc., Paris, 73, 147-154 (1871).

[25] Van Leer B., Towards the Ultimate Conservative Difference Schemes. V. A Second Order Sequel to the Godunov's Method, J. Comp. Phys., 32 (1979). 